\title{
In-piping Magnetic Actuator Capable of Inspection in a Thin Complex Pipe
}

\author{
Hiroyuki Yaguchi ${ }^{1} \&$ Kazushige Kamata ${ }^{1}$ \\ ${ }^{1}$ Faculty of Engineering, Tohoku Gakuin University, Miyagi, Japan \\ Correspondence: Hiroyuki Yaguchi, Faculty of Engineering, Tohoku Gakuin University, 1-13-1 Chuo, Tagajo, \\ Miyagi, Japan. Tel: 81-223-687-104. E-mail: yaguchi@tjcc.tohoku-gakuin.ac.jp
}

Received: June 11, 2012 Accepted: June 26, 2012 Online Published: July 26, 2012

doi:10.5539/mer.v2n2p1 URL: http://dx.doi.org/10.5539/mer.v2n2p1

\begin{abstract}
This paper proposes a magnetic actuator of a new type that exhibits a very high thrusting force and is capable of a reversible motion in a complex pipe. The magnetic actuator of the reversible type due to opening and closing of a compound material by using elongation and contraction of four shape-memory-alloy (SMA) coils was fabricated. The magnetic actuator using the difference in the frictional force between the forward and backward motion of the compound material propels in only one direction. Experimental result shows that the prototype actuator was able to climb at $38.7 \mathrm{~mm} / \mathrm{s}$ in the straight pipe when pulling the load mass of $50 \mathrm{~g}$. In addition, the measurement of an average speed carried out for two patterns of movement in a complex pipe with many curved parts and step parts. The prototype actuator was able to move in the complex pipe over the average speed of 35 $\mathrm{mm} / \mathrm{s}$. This actuator has many possible applications, including small pipe inspection.
\end{abstract}

Keywords: magnetic actuator, pipe inspection, reversible motion, shape-memory-alloy, vibration

\section{Introduction}

An actuator capable of movement in a complex pipe is required to quickly find damage for plant of chemical, heat exchanger, nuclear reactor and other such installations. The damage inside these pipes is currently inspected by x-ray or eddy current examination methods. Safety concerns have encouraged the use of such actuators in recent years for a variety of tasks, including inspection, maintenance, and cleaning. In particular, it is necessary to have an efficient method to inspect the inside of the pipes because there are approximately 10,000 straight cooling pipes in a nuclear reactor. In addition to the pipe inspection methods as mentioned above to ensure safety more, a robot capable of visually inspecting damage inside pipes is required. In the nuclear reactor in particular, a tool capable of inspection over one hundred meter inside a thin pipe of inner diameter $50 \mathrm{~mm}$ is required. A number of studies have investigated the mechanisms of a robot controlled through an electric cable to provide locomotion in a pipe using devices such as piezoelectric elements (Saito et al., 2000; Fujita et al., 2004), shape memory alloys (Reynaert et al., 1996; Kwon et al., 2005), and electromagnetic motors (Suzumori et al., 2003; Guo et al., 2005; Brunete et al., 2006; Choi et al., 2007; Miyagawa et al., 2008; Bocko et al., 2009).

However, a freely movable actuator in the complex pipe of small inner diameter with many curved parts and step parts has not been addressed yet. The authors previously proposed (Yaguchi et al., 2010) a novel globular magnetic actuator that provides propulsion by means of the inertia force of a mass-spring system excited by an electromagnetic force. There are potential problems in the design of the actuator, and it is necessary to increase the propulsion force of the total system in order to install additional components, such as many CCD-cameras and LED-lights. This paper proposes a magnetic actuator of a new type capable of inspection in the complex pipe. We also confirmed a reversible motion of the magnetic actuator due to on-off control of a direct current which is electrified into four shape-memory-alloy (SMA) coils attached at a body. Experimental result shows that the maximum vertical upward speed of the magnetic actuator capable of the reversible motion is $62.5 \mathrm{~mm} / \mathrm{s}$ when inner diameter of the pipe is $50 \mathrm{~mm}$. In addition, this actuator is able to move freely in the complex pipe with many curved parts and step parts. The results hold great promise for the creation of highly mobile actuators capable of the reversible motion in the complex pipes with small diameters.

\section{Magnetic Actuator Capable of Freely Movement in the Complex Pipe}

Figure 1(a) is a basic diagram of the magnetic actuator capable of movement in the complex pipe. The magnetic 
actuator is composed of a propulsion module as shown in Figure 1(b), the four shape-memory-alloy (SMA) coils, two layer compound material A and B, eight copper conductors attached at the compound materials and the acrylic frame. The SMA coil is shape of a spring type with an outer diameter of $0.4 \mathrm{~mm}$ and length of $14 \mathrm{~mm}$ when it was completely contracted. The outer diameter of a SMA wire to form the SMA coil is $0.15 \mathrm{~mm}$. This SMA coil has an electrical resistance of $400 \Omega$ per unit length. Accordingly, an electrical resistance of this SMA coil is $5.6 \Omega$. The compound material to support the actuator in the pipe is composed of a natural rubber and a silicone rubber. This is $95 \mathrm{~mm}$ in length, thickness of the natural rubber is $1.5 \mathrm{~mm}$ and thickness of the silicone rubber is $2 \mathrm{~mm}$, respectively. The two compound materials were combined by the flexible natural rubber bar. The propulsion module of the actuator consists of the four permanent magnets, a four translational springs and a four electromagnets. The magnetic actuator has an acrylic frame with diameter of $35 \mathrm{~mm}$ and thickness of $2 \mathrm{~mm}$. To promote propulsion properties of the actuator, twin type vibration system by combination the two vibration models was used in this paper. The two vibration bodies 1,3 and 2, 4 were connected by using an iron material with height of $4.5 \mathrm{~mm}$, width of $12 \mathrm{~mm}$, and thickness of $2 \mathrm{~mm}$. The two vibration systems were connected by magnetic circuit as shown in Figure 1(b). The permanent magnet was attached on the spring, respectively. The mass-spring system composed due to the electromagnet and the translational spring was adhered to the acrylic frame. The translational spring is stainless steel type with an outer diameter of $7 \mathrm{~mm}$, free length of $12 \mathrm{~mm}$, and a spring constant $\mathrm{k}=1595 \mathrm{~N} / \mathrm{m}$. The permanent magnet is cylindrical NdFeB and is magnetized in the axial direction. This is $7 \mathrm{~mm}$ in diameter and height is $3 \mathrm{~mm}$. The surface magnetic flux density measured by using a tesla meter was $345 \mathrm{mT}$. The electromagnet consists of an iron core $2.4 \mathrm{~mm}$ in diameter with 504 turns of 0.12 $\mathrm{mm}$ diameter copper wire. The electrical resistance of the electromagnet is $10.4 \Omega$ when the two electromagnets were connected by magnetic circuit. The surface magnetic flux density at both edges of the electromagnet module measured by using the tesla meter was $42.4 \mathrm{mT}$ when an alternating current of the effective value $0.2 \mathrm{~A}$ was electrified into the electromagnet. The gap between the electromagnet and the permanent magnet in the static condition is $3 \mathrm{~mm}$, respectively. This actuator converts the mechanical resonance energy of the propulsion module stimulated by the AC electrical power source into locomotive power. Figure 2 shows structure of the magnetic actuator capable of the reversible motion. The two CCD-cameras and the four LED-lights were attached to the acrylic frame to inspect inside the complex pipes. The total mass of the magnetic actuator is 27.2 g. Photo1 shows the photograph of the magnetic actuator.

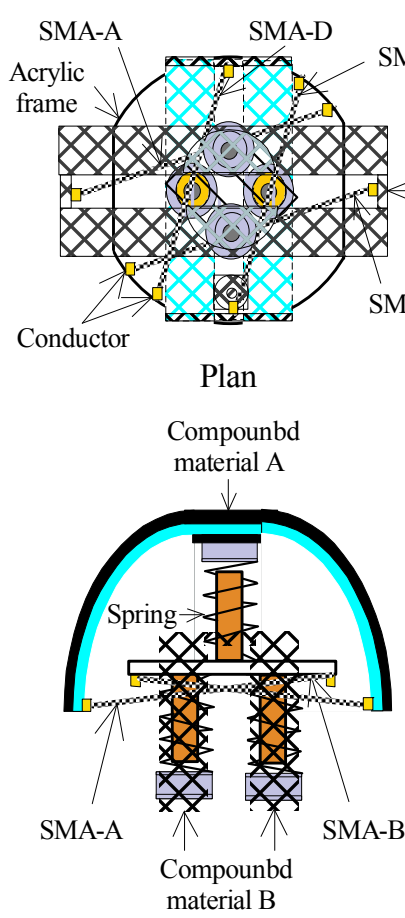

Front view

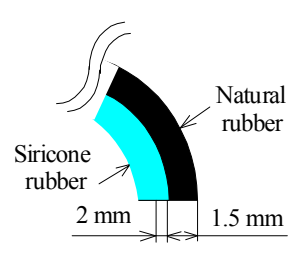

Compound material
SMA-B

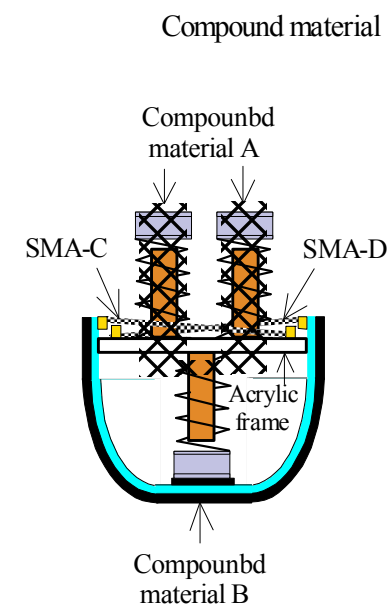

Side view
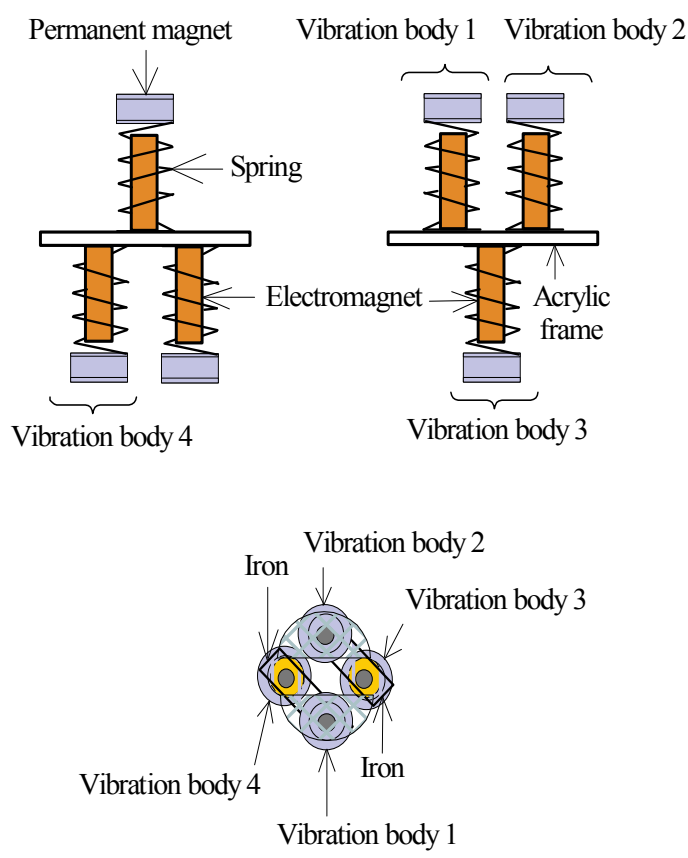

(a) Outline of the magnetic actuator

(b) Detail of the propulsion module

Figure 1. Structure of the magnetic actuator capable of the reversible motion 


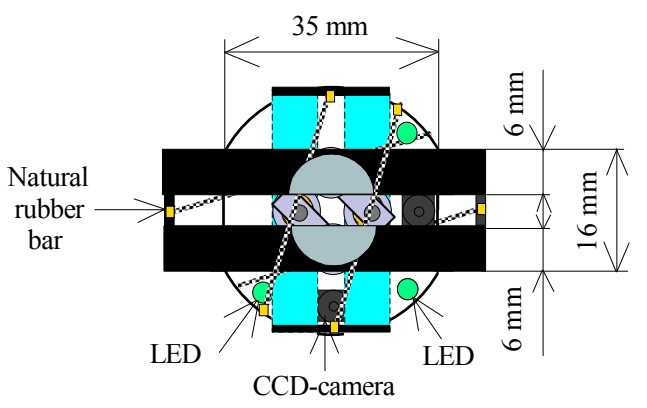

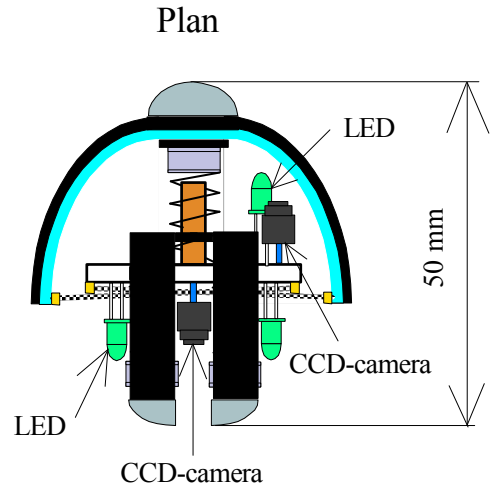

Front view

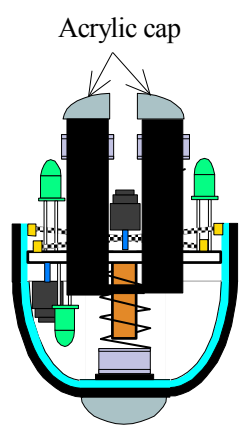

Sideview

Figure 2. Structure of the magnetic actuator capable

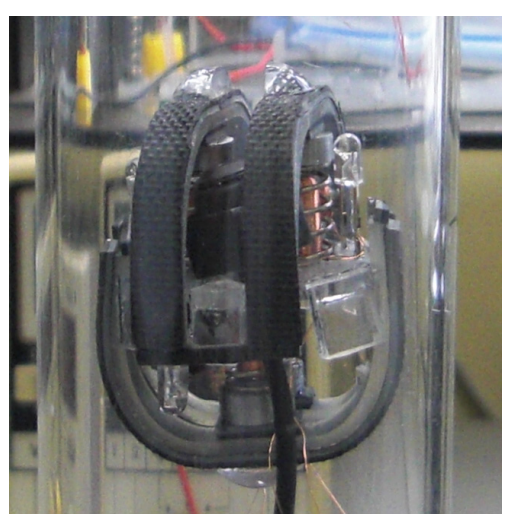

Photo 1. Photograph of the magnetic actuator of the reversible motion

\section{Vibration Mode of Magnetic Actuator}

The mass-spring system can be approximated as a three-degree-of-freedom model, as shown in Figures 3(a). In these figures, the sum of the mass of the acrylic frame, the four electromagnets, the two iron plates, the four LED-lights, the two CCD-cameras, the vibration body 5 and also the four SMA coils is M, and the sum of the mass of the two acrylic caps, the two permanent magnets, and the two compound materials is $\mathrm{m}$. If the masses are treated as point masses and displacements $x_{i}(i=1,2,3)$ are measured as shown in Figure 3(a), then the equations of translational motion can be written as follows: 


$$
\left.\begin{array}{c}
m \frac{d^{2} x_{1}}{d t^{2}}+2 k\left(x_{1}-x_{2}\right)=0, m \frac{d^{2} x_{3}}{d t^{2}}-2 k\left(x_{2}-x_{3}\right)=0 \\
M \frac{d^{2} x_{2}}{d t^{2}}-2 k\left(x_{1}-2 x_{2}+x_{3}\right)=0
\end{array}\right\}
$$

The solution of Equation (1) yields the eigenvibration mode and the eigenfrequency of the mass-spring system. Here, $M=23 \mathrm{~g}, \mathrm{~m}=1.4 \mathrm{~g}$. The first eigenfrequency is $\mathrm{f}_{1}=0 \mathrm{~Hz}$, and the second eigenfrequency is $\mathrm{f}_{2}=169.8 \mathrm{~Hz}$. The third eigenfrequency is $f_{2}=180 \mathrm{~Hz}$. The first vibration mode is the rigid mode. Figures 3(b) and 4(c) illustrate the second and third vibration modes of the model in the state in which the actuator is not inserted in the pipe. As a driving mode of the actuator, the second mode was used in this experiment.

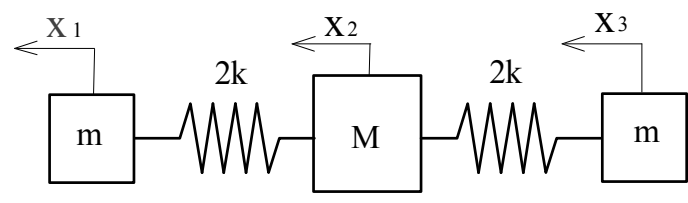

(a) Mass-spring model

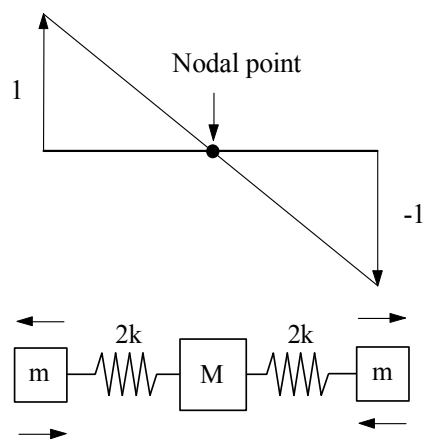

(b) Second mode

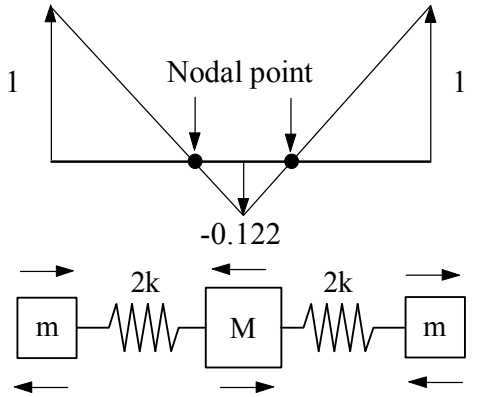

(c) Third mode

Figure 3. Three-degrees-of-freedom model

\section{Principle of Locomotion}

The vibration displacement of the body mass $M$ due to constraint of the compound material becomes smaller when the magnetic actuator was inserted in the pipe. Therefore, we think that the actuator moves by using the inertia force of the vibrating mass $\mathrm{m}$. The principle of locomotion is as follows:

1) As shown in Figure 4 (a), the supporting force of the compound material is not changed due to inertia force of a vibrating mass $\mathrm{m}$ when the mass $\mathrm{m}$ vibrates in the direction of coordinate $\mathrm{z}$. The magnetic actuator is able to slide when the inertia force of the vibrating mass $\mathrm{m}$ is bigger than a frictional force between the compound material and wall of the pipe.

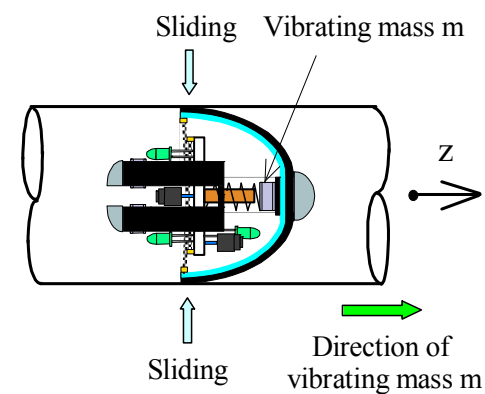

(a) Case of movement

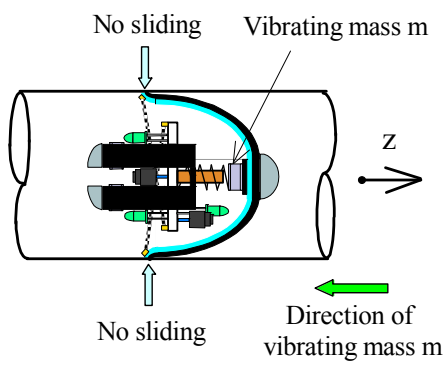

(b) Case of no movement

Figure 4. Principle of locomotion 
2) On the other hand, tip of the compound material as shown in Figure 4 (b) is transformed, and locked in wall inside the pipe when the mass $\mathrm{m}$ moves in the opposite direction of coordinate $\mathrm{z}$. In this case, the frictional force between the compound material and wall of the pipe becomes quite big compare with inertia force of the vibrating mass $\mathrm{m}$. Therefore, the magnetic actuator is not able to move.

3) The frictional force between the compound material and wall of the pipe inside alternately changes during one period of the vibration. As a result, actuator is able to move only in the one direction of coordinate $z$.

\section{Principle of Reversible Motion}

Figure 5 shows outline of the reversible motion. The SMA coils are heated when the direct current is electrified into the SMA coils by connecting to the DC power supply. Therefore, the SMA coils contract. The compound material B is completely closed due to contraction of the two SMA-C and SMA-D coils when the direct current is electrified into the two SMA-C and SMA-D coils. The direct current was limited to $0.2 \mathrm{~A}$ per one coil by considering the influence of the heat. Then, the generating force measured by using a force gauge was $0.2 \mathrm{~N}$. The magnetic actuator is able to move in direction of straight up as shown in Figure 5(a).

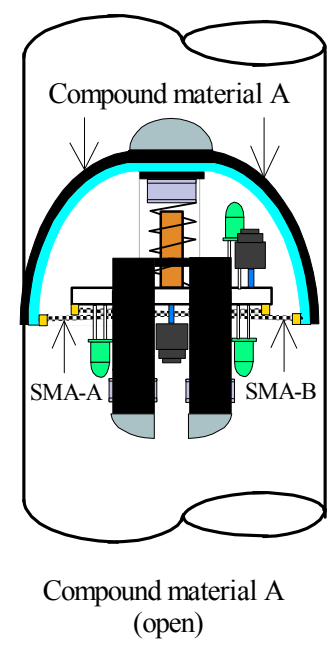

(a) Straight up

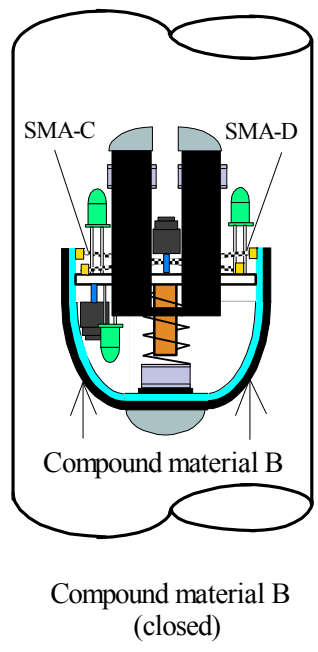

(closed)

Figure 5. Method of the reversible motion due to control of the input current into the SMA coils

On the other hand, the compound material A is completely closed when the direct current is electrified into the two SMA-A and SMA-B coils. The SMA-C and SMA-D coils are cooled by air-cooling when supply of the direct current into the two SMA-C and SMA-D coils is stopped. After a few second, the compound material B opens by elastic restoring force of the material itself. As a result, the magnetic actuator is able to move in direction of straight down in Figure 5(b).

\section{Basic Locomotion Characteristics}

An experimental test was conducted by using an apparatus as shown in Figure 6. The first resonance frequency of the magnetic actuator measured by using experimental apparatus was $167 \mathrm{~Hz}$.

Figure 7 shows the relation between the tilt angle $\alpha$ of the straight pipe as shown in Figure 6 and the speed of the magnetic actuator with respect to the straight pipe with inner diameter of $45 \mathrm{~mm}$ and $50 \mathrm{~mm}$. The tilt angle was varied from $-90^{\circ}$ (straight down) to $90^{\circ}$ (straight up). In this figure, the maximum vertical upward speed was $62.5 \mathrm{~mm} / \mathrm{s}$ when inner diameter of the pipe was $50 \mathrm{~mm}$ and input current in the actuator was $0.32 \mathrm{~A}$. For the pipe with inner diameter of $50 \mathrm{~mm}$, the speed when moving straight down was about 3.5 times that when moving straight up. This indicates that the mass of the actuator was a factor, suggesting that further mass reduction of the actuator is desirable. 


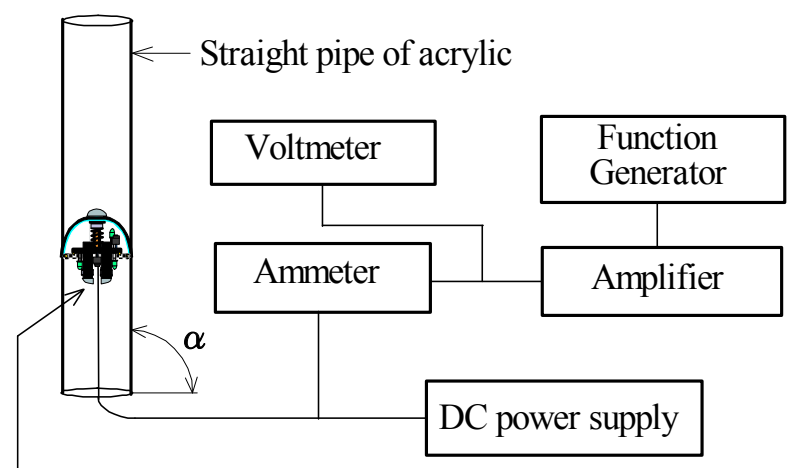

Magnetic actuator

Figure 6. Experimental apparatus

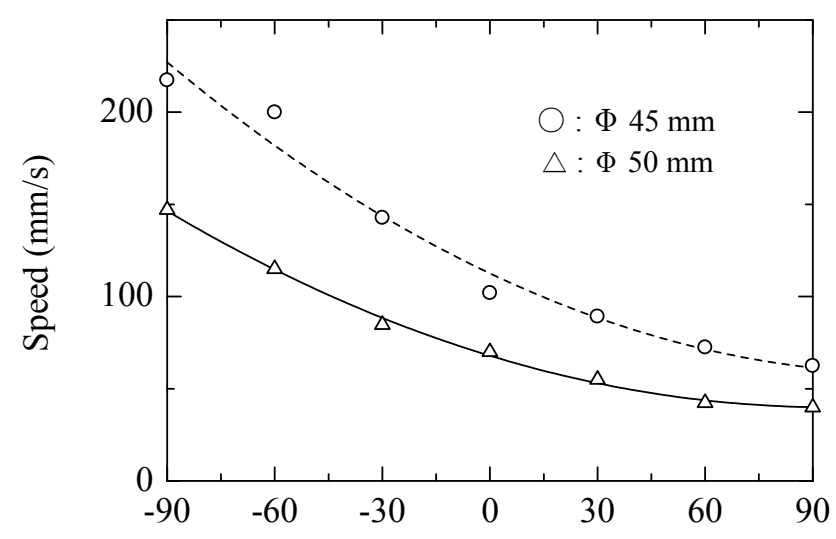

Tilt Angle (deg)

Figure 7. Relationship between tilt angle and speed

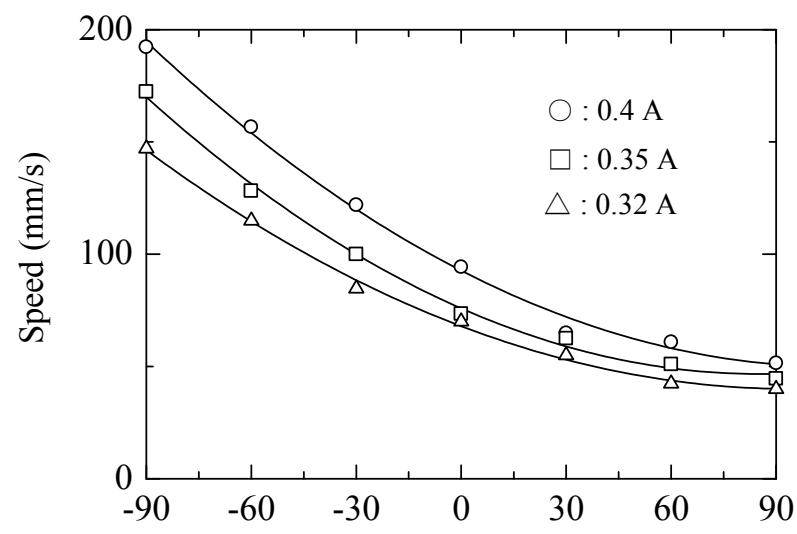

Tilt Angle (deg)

Figure 8. Relationship between tilt angle and speed 


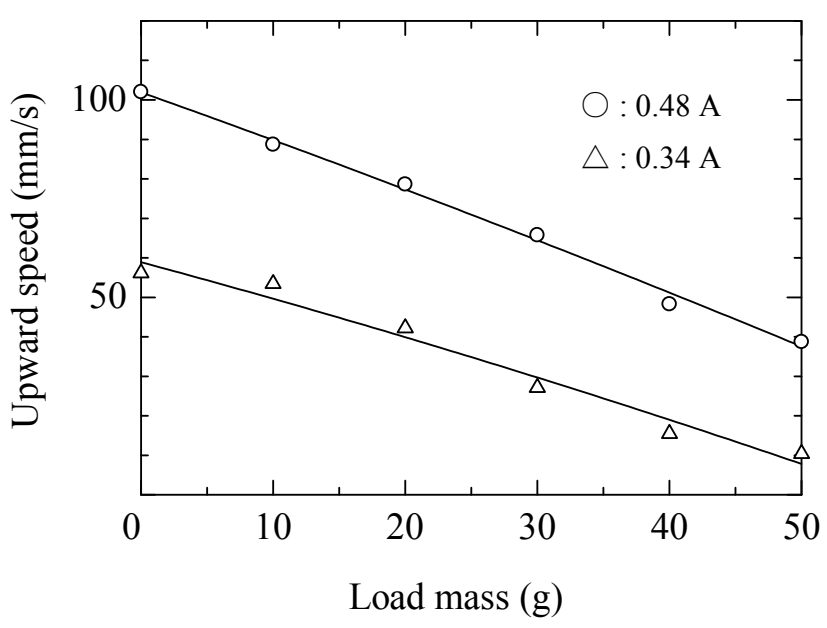

Figure 9. Relationship between load mass and vertical upward speed

Figure 8 shows the relation between the tilt angle of the straight pipe and the speed of the magnetic actuator with respect to the straight pipe with inner diameter of $45 \mathrm{~mm}$ when input current was changed $0.32 \mathrm{~A}, 0.35 \mathrm{~A}$, and 0.4 A.

Figure 9 shows the relation between the load mass and the vertical upward speed for the straight pipe with inner diameter of $45 \mathrm{~mm}$ when input current was changed $0.34 \mathrm{~A}$ and $0.48 \mathrm{~A}$. This figure indicates that the magnetic actuator was able to climb at $38.7 \mathrm{~mm} / \mathrm{s}$ when pulling the load mass of $50 \mathrm{~g}$ and inner diameter of $45 \mathrm{~mm}$, a moderately high performance.

\section{Moving Properties in Complex Pipe}

Figure 10 shows detail of the complex pipe with two curved part and two step parts of $2.5 \mathrm{~mm}$ in the pipe. In addition, the diameter of the curved part and that of an entrance part at this pipe are different. This magnetic actuator was required ability of movement over the wide range of inner diameter from $43 \mathrm{~mm}$ to $48 \mathrm{~mm}$. The complex pipe with such configuration is often used for a joint of a water supply, a cooling pipe and the heat exchanger. The distance of the measurement was set as $190 \mathrm{~mm}$ with the two curved parts and the three straight parts. The measurement of an average speed carried out for two moving patterns in the horizontal direction and vertical direction as shown in Figure 11. Compared to pattern I, in pattern II, the body of the magnetic actuator in the pipe is rotated 90 degrees.

By combination of the mass-spring system with the excellent permanent magnets and the compound material with low stiffness, the magnetic actuator proposed in this paper was able to move easily in the complex pipe with many curved parts and step parts.

Table 1 shows the average speed for the two patterns of movement about the actuator measured in the horizontal and vertical direction. The experimental results show that, for both patterns, the average speed of the magnetic actuator exceeds $43 \mathrm{~mm} / \mathrm{s}$.

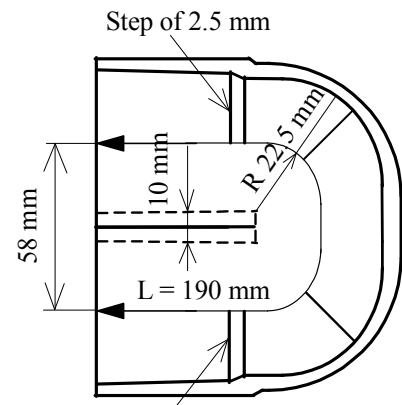

Step of $2.5 \mathrm{~mm}$

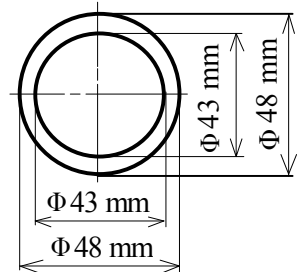

Detail of section A-A'

Figure 10. Detail of the complex pipe 


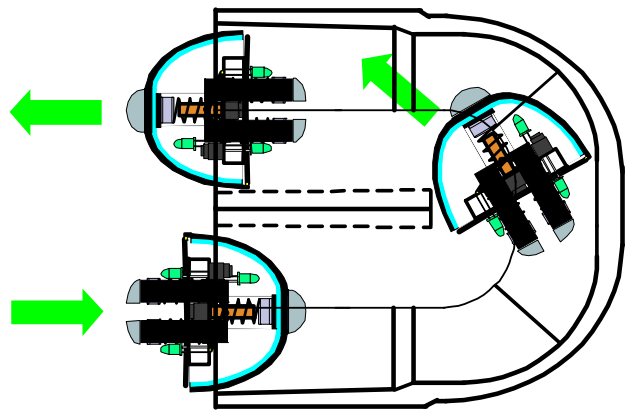

(a) Pattern A

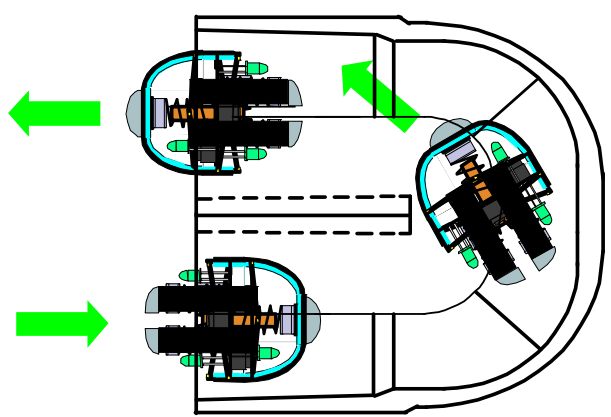

(b) Pattern B

Figure 11. Two moving patterns in the complex pipe

Table 1. Average speed for two patterns of movement

\begin{tabular}{lcccc}
\hline & \multicolumn{2}{c}{ Horizontal direction } & \multicolumn{2}{c}{ Vertical direction } \\
\cline { 2 - 5 } & Pattern I & Patern II & Pattern I & Patern II \\
\cline { 2 - 5 } $\begin{array}{l}\text { Average speed of } \\
\text { actuator }(\mathrm{mm} / \mathrm{s})\end{array}$ & 63.5 & 57.3 & 43.8 & 43.2 \\
\hline
\end{tabular}

Finally, we inspected in the complex pipe with many curved parts and straight parts by using this magnetic actuator. In the nuclear reactor in particular, the inspection in the pipe with T-junction is required. We have to attempt establish of the principle of locomotion in the complex pipes with T-junction. If the power properties of the propulsion module by modification of the magnetic circuit can be inproved, it certainly seems possible to extend the range of movement to several hundred meters.

\section{Conclusion}

A magnetic actuator capable of freely movement in a complex pipe has been proposed and tested. Further, we proposed a reversible motion of the magnetic actuator due to on-off control of input current into four SMA coils. The experimental result shows that the magnetic actuator was able to climb at $38.7 \mathrm{~mm} / \mathrm{s}$ when pulling the load mass of $50 \mathrm{~g}$ and inner diameter of $45 \mathrm{~mm}$.

The measurement of an average speed carried out for two moving patterns in the complex pipe. The result demonstrated that the magnetic actuator was able to move in the complex pipe over the average speed of 43 $\mathrm{mm} / \mathrm{s}$. In addition, the actuator was able to move stably in the straight pipe and the elbow pipe without rotational motion of a body. This magnetic actuator has many possible applications, including inspection by the actuator with a CCD-camera and maintenance of the pipes. Future research will be directed toward these goals.

\section{Acknowledgements}

This work was supported in part by Grants-in-Aid for Scientific Research (C) (11010738), Japanese Ministry of Education (2011-2014).

\section{References}

Bocko, J., Kelemen, M., Kelemenova, T., \& Jezny, J. (2009). Wheeled Locomotion inside Pipe. Bulletin of Applied Mechanics, 5(8), 34-36.

Brunete, I., Torres, J., Hernando, M., \& Gambao, E. (2006). A 2 DoF Servomotor-based Module for Pipe Inspection Modular Micro-robots. Proc. International Conference on Intelligent Robots and Systems. http://dx.doi.org/10.1109/IROS.2006.281898

Choi, H., \& Roh, S. (2007). In-pipe Robot with Active Steering Capability for Moving Inside of Pipelines. Bioinspiration and Robotics Walking and Climbing Robots, 23, 375-400.

Fujita, J., Shiraogawa, Y., Yamamoto, S., \& Kato, T. (2004). Study of Mobile Mechanism with Elastic Fibers by Using Vibration. Trans. Japan Soc. Mec. Eng., 70(698), 22-26. http://www.jsme.or.jp/publish/rbj0410c.htm

Guo, S., \& Pan, Q. (2005). A Paddling Type of Microrobot in Pipe. Proc. IEEE/RSJ International Conference on 
Intelligent Robots and Systems, 2265-2270.

Kwon, J., Park, S., Kim, B., \& Park, J. (2005). Bio-Material Property Measurement System for Locomotive Mechanism in Gastro-Intestinal Tract. Proc. IEEE International Conference on Robotics and Automation, 1315-1320. http://dx.doi.org/10.1109/ROBOT.2005.1570295

Miyagawa, T., \& Iwatski, N. (2008). Moving Chacteristics in Bent Pipes of In-Pipe Mobile Robot with Wheel Drive Mechanism using Planetary Gear Drive. Journal of the Japan Society for Precision Engineering, 74(12), 1346-1350. http://dx.doi.org/10.2493/jjspe.74.1346

Reynaerts, D., Peilw, J., \& Brussel, H. (1996). Design of a Shape Memory Actuated Gastrointestinal Intervention System. Proc. Actuator, 96.

Saito, H., Sato, K., Kudo, K., \& Sato, K. (2000). Fundamental Study of Mover Travel inside a Small Diameter Pipe. Trans. Japan Soc. Mec. Eng., 66(641), 346-353. http://www.jsme.or.jp/publish/rbj0001c.htm

Suzumori, K., Wakimoto, S., \& Tanaka, M. (2003). In pipe Inspection Micro Robot Adaptable to Changes in Pipe Diameter. Proc. IEEE International Conf. Robotics and Automation, 2735-2740.

Yaguchi, H., \& Sato, N. (2010). Globular Magnetic Actuator Capable of Freely Movement in a Complex Pipe. IEEE Trans. Magn., 46(6), 1350-1355. http://dx.doi.org/10.1109/TMAG.2010.2040712 